\title{
Pathohistological and Immunohistochemical Analysis of Primary Invasive Ductal Breast Carcinoma with Signet-Ring Cell Differentiation- Differential Diagnosis, Prognosis and Complex Treatment
}

\author{
Lena Marinova $^{* 1}$, Bistra Yordanova ${ }^{2}$, Nikolay Evgeniev ${ }^{3}$, Dorothea Malinova ${ }^{4}$ \\ ${ }^{1}$ Department of Radiotherapy, Complex Oncology Center - Ruse, ${ }^{*}$ Corresponding authors \\ Bulgaria \\ Lena Marinova, Department of Radiotherapy, Complex Oncology \\ ${ }^{2}$ Department of Clinical Pathology, Complex Oncology Center - \\ Center - Ruse, Bulgaria \\ Ruse, Bulgaria \\ Submitted:08 March2021;Accepted:15 March2021;Published:22 March 2021
}

${ }^{3}$ Medical Oncology Department, Complex Oncology Center - Ruse, Bulgaria

${ }^{4}$ Department of General and Clinical Pathology, Medical University, Varna, Bulgaria

Citation: Lena Marinova, Bistra Yordanova, Nikolay Evgeniev, Dorothea Malinova (2021). Pathohistological and immunohistochemical analysis of primary invasive ductal breast carcinoma with signet-ring cell differentiation-Differential diagnosis, prognosis and complex treatment. Medical \& Clinical Research 6(3): 464-469.

\begin{abstract}
Primary signet ring cell carcinoma (PSRCC) of the breast is a rarely diagnosed neoplasm. We present a 76-year-old woman with a tumor formation in the left mammary gland, who has been self-medicating for a year. Pathohistological and immunohistochemical analysis proved rare primary invasive ductal carcinoma with focal (over 90\%) signet ring cell differentiation, size $4 \mathrm{~cm} / 3.5 \mathrm{~cm} / 2 \mathrm{~cm}$, moderately differentiated (G2). Complex oncological treatment, including radical mastectomy with axillary dissection, 6 courses of systemic adjuvant chemotherapy, radiotherapy of the chest wall and regional lymph nodes with TD 46 Gy and antiestrogenic hormone therapy, was performed. The diagnosis and the differential diagnosis of this rare tumor require precise pathohistological and immunohistochemical analysis. The prognosis and complex treatment depend on the clinical stage, hormonal and HER2 status. In locally advanced PSRCC of the breast with moderately differentiation, the combination of surgery, systemic chemotherapy, postoperative radiotherapy and antiestrogenic hormone therapy achieves long-term local tumor control without distant metastases for nearly two years.
\end{abstract}

Keywords: Primary Signet Ring Cell Carcinoma of the Breast, Pathohistological Analysis, Immunohistochemical Analysis, Differential Diagnosis, Prognosis, Complex Treatment

\section{Introduction}

The definition of a signet ring cell (SRC) is a cell with a large vacuole, with a cytoplasm containing mucin and displaces nucleus [1]. Signet ring cell carcinoma (SRCC) is a rare form of highly malignant adenocarcinoma, that produces mucin [2, 3]. It is an epithelial malignancy characterized by the histologic appearance of SRCs. Primary SRCC tumors are most often found in the glandular cells of the stomach and less frequently in the prostate, breast, gallbladder, urinary bladder, colon, ovarian stroma, testis and pancreas [4-9]. In this article we present a clinical case of a rare signet ring cell breast carcinoma (SRCBC). Our main goal is to present the importance of pathohistological and immunohistochemical analysis in the diagnosis of primary
SRCBC, with an emphasis on the prognosis and differential diagnosis.

Clinical Case - This is a 76-year-old woman who noticed a growing painless formation in the left breast. After a year of selfmedication, she went to the doctor. So in June 2019 the disease has been diagnosed.

From the research - Mammography - Left mammary gland with solid formation, vaguely demarcated in the upper lateral quadrant. ACR density from $2 \%$ to $50 \%$, asymmetric density increased in the left mammary gland.

Ultrasound of the left mammary gland - A hypoechoic zone 
of $30 / 26 \mathrm{~mm}$ at the site of formation with blurred borders to the surrounding parenchyma, positive Doppler signals in the periphery with strong subsequent echocardiography, is reported.

Left axillary area - a package of enlarged lymph nodes, the largest with a maximum diameter of $22 / 13 \mathrm{~mm}$ with a hypoechoic center with pronounced hypoechoic periphery.

CT of the chest and abdomen with intravenous contrast - No evidence of distant metastases.

Preoperative local status -Visible retraction of the skin in the upper lateral quadrant and the nipple. A solid immobile tumor with dimensions of about $3 \mathrm{~cm}$ is palpated. Left axilla - enlarged lymph node up to $2 \mathrm{~cm}$ with a firm consistency. Right mammary gland and right axilla - without pathological changes.

Intraoperatively - A modified radical mastectomy a modo Pirogov on the left mammary gland with removal of the pectoral fascia, as well as axillary lymph dissection at the I axillary level and partial at the II level was performed. Some of the lymph nodes at axillary level II due to metastatic tumor infiltration of the axillary vein were not removed. Small clinically metastatic hard lymph nodes at axillary level III and subclavicular were palpated.

Histological result - Macroscopic - Material from mastectomy of the left mammary gland with dimensions $29 \mathrm{~cm} / 18 \mathrm{~cm} / 5$ $\mathrm{cm}$. Leather fragment $22 \mathrm{~cm} / 11 \mathrm{~cm}$ with retraction of the nipple. Tumor measuring $4 \mathrm{~cm} / 3.5 \mathrm{~cm} / 2 \mathrm{~cm}$ with whitish color and cartilage density.

Microscopic - From the Tumor Formation - Invasive ductal carcinoma with focal signet ring cell (SRC) differentiation, moderately differentiated (G2). Significant cellular polymorphism, pronounced anisonucleosis with hyperchromasia is reported (Figure 1/A,B). Mamila - without pathological changes. Twenty axillary lymph nodes, of which nineteen with metastases. Adipose tissue with tumor deposits. Resection lines - without tumor infiltration.

Immunohistochemistry (IHC)- Estrogen receptor (ER) - strongly positive reaction $(3+5=8)$ (Figure $2 / \mathrm{A})$; Progesterone receptor (PR) - strongly positive reaction $(3+4=7$ ) (Figure $2 / B$ ); HER2 - negative reaction (Figure 2/C); E-cadherin- diffuse positive reaction in tumor cells (Figure 3/A); gross cystic disease fluid protein (GCDFP)- diffuse moderately positive reaction in tumor cells (Figure 3/B); CK7 - diffuse strongly positive reaction in tumor cells (Figure 3/C); Alcian blue (PAS) staining positivizes intracytoplasmic mucin in tumor cells (Figure 4/A,B); thyroid transcription factor-1 (TTF1)-negative reaction (Figure 5/A); caudal type homeobox 2 (CDX2)-negative reaction (Figure 5/B); CK20-negative reaction (Figure 5/C).

Histological Diagnosis - Invasive ductal carcinoma with focal (over $90 \%$ ) signet ring cell differentiation, size $4 \mathrm{~cm} / 3.5 \mathrm{~cm} / 2 \mathrm{~cm}$, moderately differentiated (G2). Presence of perineural invasion and lymphovascular tumor emboli. Mamila- uninvolved. Mastectomy with clean resection lines. Metastases in nineteen axillary lymph nodes, of the twenty lymph nodes examined. / pT2 N3a Mx G2.
The Oncology Committee assessed the patient for complex treatment, including postoperative systemic chemotherapy (Ch), radiotherapy (RT) and endocrine therapy. After 6 courses of Ch with Docetaxel Actavis, we performed Intensive Modulated Radiotherapy (IMRT) using the VMAT technique with clinical target volume (CTV) involving the left chest wall with the operative scar and regional lymph nodes (axillary, supraclavicular and parasternal) up to total dose (TD) 46 Gy with daily dose (DD) 2 Gy (Figure 6). Due to cardiac comorbidity involving atrial fibrillation, the TD was estimated not to exceed 46 Gy. After the RT, the patient received antiestrogenic endocrine therapy for 2 years. In November 2020, on a control CT 6 months after the completion of RT, suspected metastases of osteoplastic changes of the vertebral bodies Th11 and L4 were detected (Figure 7) which were rejected by bone scintigraphy. Without significant dynamics, the same changes are observed on CT after 3 months in January 2021 (Figur 8). The patient is in good general condition 20 months after the complex treatment, without recurrence and distant metastases.

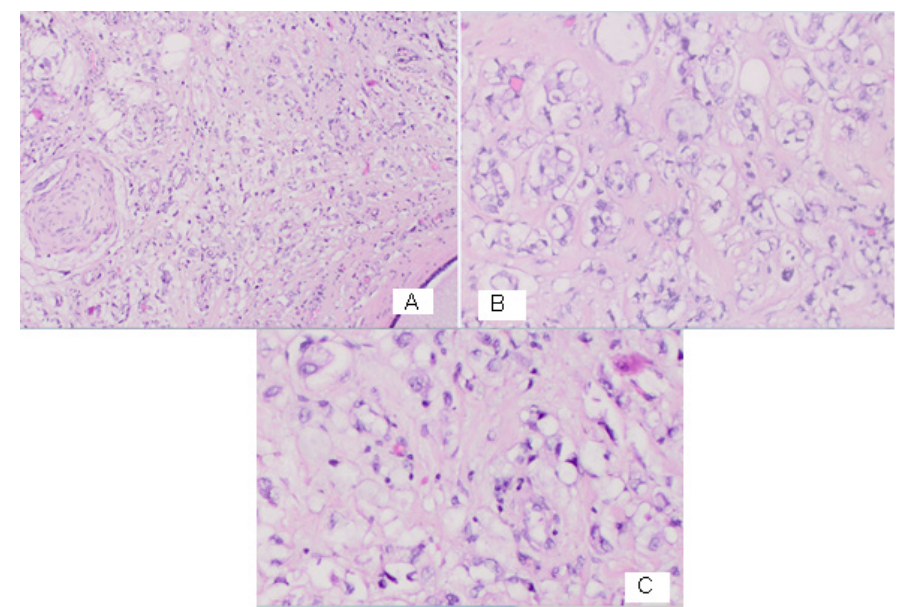

Figure 1: Pathohistological characteristics of invasive ductal carcinoma with focal signet ring cell differentiation, moderately differentiated (G2) A/ H\&E x20; B/C Polymorphic signet ring carcinoma cells with anisonucleosis and hyperchromasia. A nucleus pressed against the cell periphery as a result of diffusely filling the cytoplasm mucin. H\&E x100.

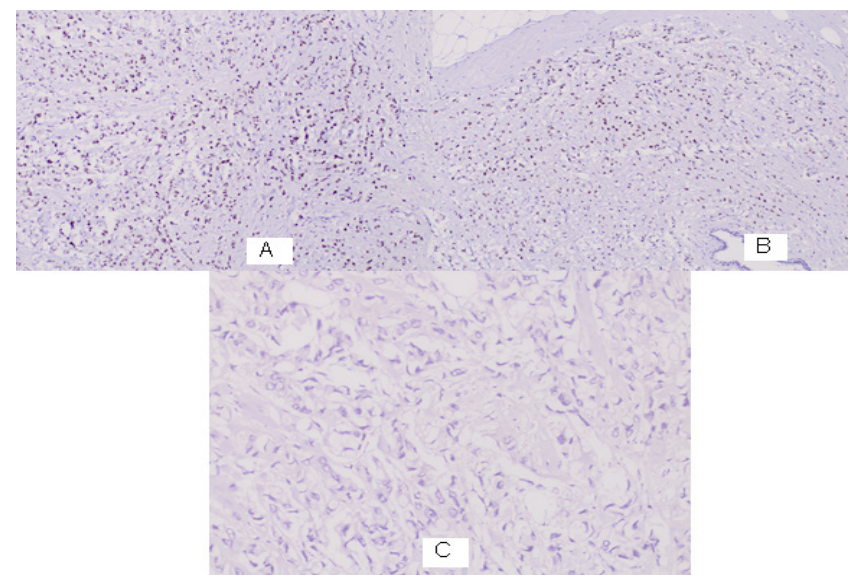

Figure 2: Photomicrography of signet-ring cell breast carcinomaImmunohistochemical analysis-A/ Estrogen receptor (ER) - 
strongly positive IHC reaction $\mathrm{x} 20$; B/ Progesterone receptor (PR) - strongly positive IHC reaction x 20; C/ HER2 - negative IHC reaction $\mathrm{x} 20$

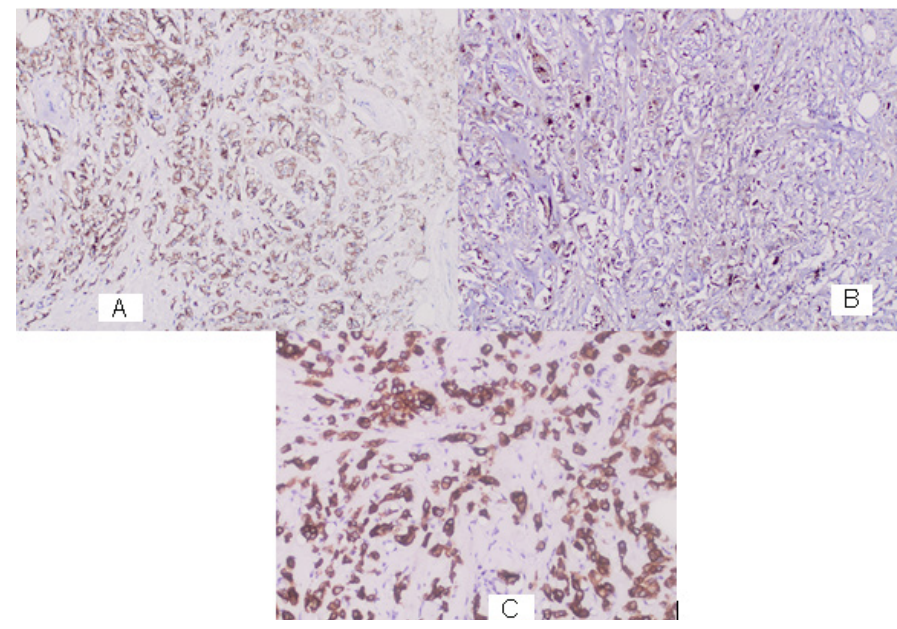

Figure 3: Photomicrography of positive immunohistochemical analysis in tumor cells of signet-ring cell breast carcinoma $-\mathrm{A} /$ E-cadherin- diffuse positive expression x 20; B/ GCDFP- diffuse moderately positive expression x $20 ; \mathrm{C} / \mathrm{CK} 7$ - diffuse strongly positive expression $\mathrm{x} 20$

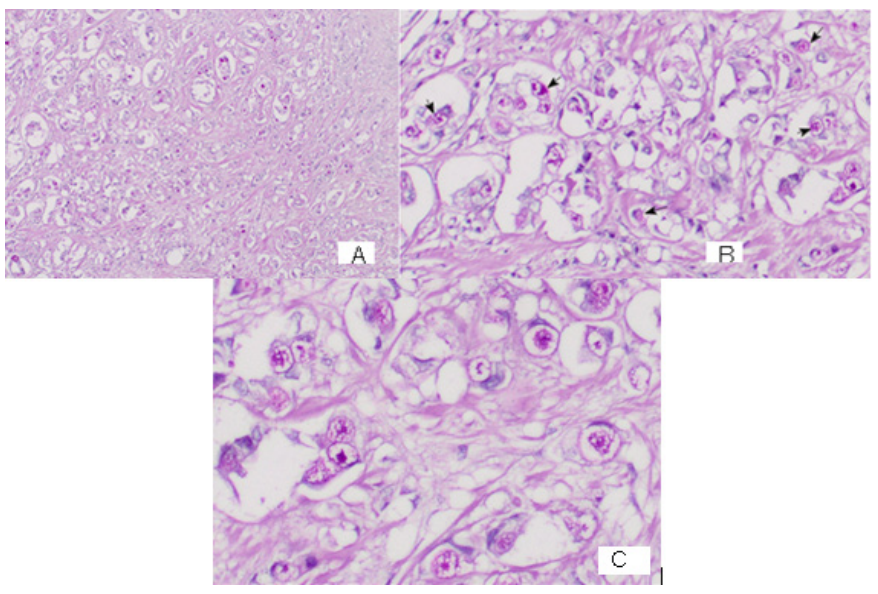

Figure 4: Photomicrography of signet-ring cell breast carcinoma with Alcian blue (PAS) staining-A/ PAS staining demonstrates the presence of cellular intracytoplasmic mucin x 20; B/ Black arrows represent intracytoplasmic mucin in tumor cells that the nucleus pushes into the cell periphery x 100; C/ PAS staining x 400 .

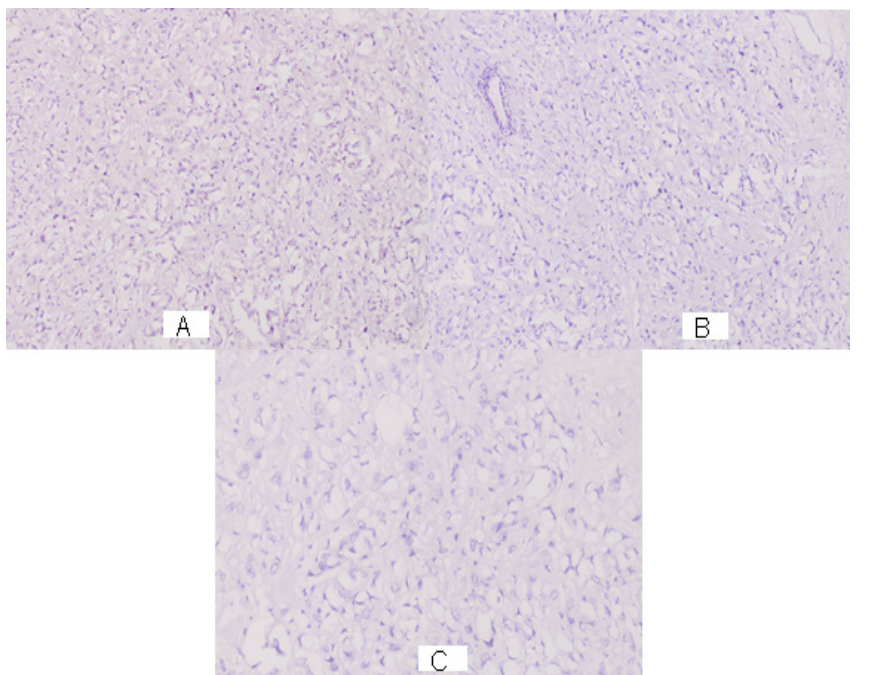

Figure 5: Photomicrography of negative immunohistochemical analysis in tumor cells of signet-ring cell breast carcinoma - A/ Negative TTF1 expression x 20; B/ Negative CDX2 expression $\mathrm{x}$ 20; C/ Negative CK20 expression x 20
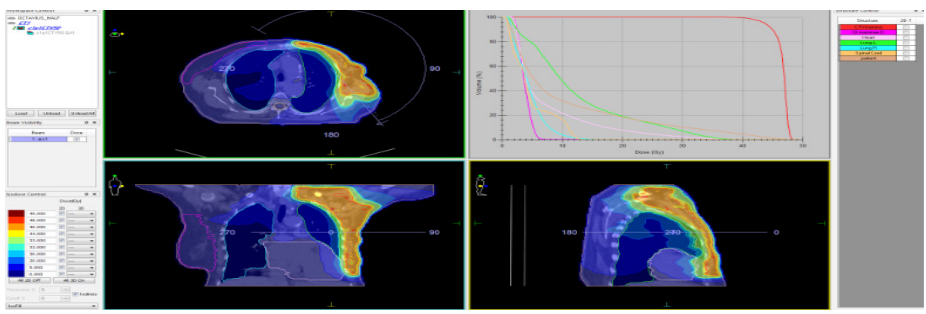

Figure 6: Intensive modulated radiotherapy (IMRT) using the VMAT technique with clinical target volume (CTV) involving the left chest wall with the operative scar and regional lymph nodes (axillary, supraclavicular and parasternal) up to TD 46 Gy with DD 2 Gy.

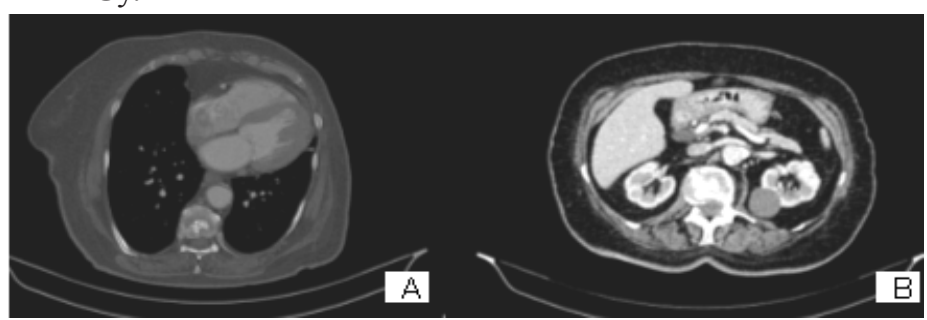

Figure 7: $\mathrm{A} / \mathrm{CT}$ of the chest / bone window - Osteoplastic lesion of the Th11 vertebral body.; B/ CT of the abdomen - A parenchymal 
cyst $33 \mathrm{~mm}$ in diameter in the left kidney and osteoplastic lesion of the L4 vertebral body. Without pathological changes in the liver and paraaortic lymph nodes.

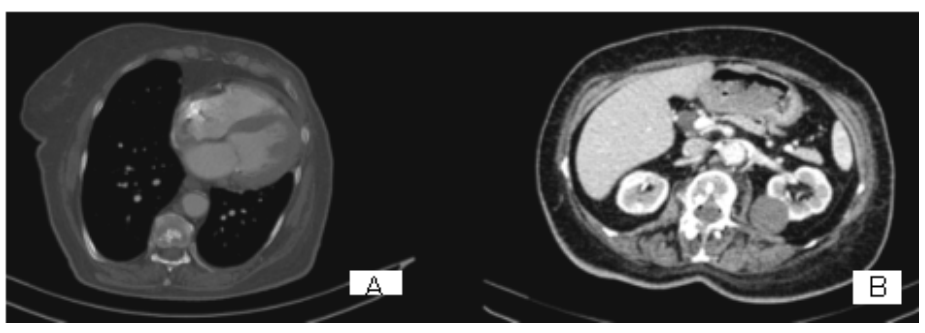

Figure 8: A/CT of the chest / bone window - Osteoplastic lesion of the Th11 vertebral body without dynamics; B/CT of the abdomen - A parenchymal cyst $35 \mathrm{~mm}$ in diameter in the left kidney and osteoplastic lesion of the L4 vertebral body without dynamics. Without pathological changes in the liver and paraaortic lymph nodes.

\section{Discussion}

Primary signet ring cell breast carcinoma (PSRCBC) was first described by Saphir in 1941 as a type of mucinous carcinoma and since then only few cases have been reported in English literature [10-15]. The prevalence of signet-ring features has ranged from 2 to $4.5 \%$ of total breast cancers $[12,14,16]$. In 1976, Steinbrecher and Silverberg reported five cases of rare carcinoma of the breast, characterized by the presence of numerous cells containing intracellular mucin, without large amounts of extracellular mucins seen in colloid carcinoma of the breast [17]. Signet ring cell breast carcinoma (SRCBC) can be divided into primary and metastatic tumors [3, 18]. The general definition of PSRCBC is ductal carcinoma with cells resembling gastric carcinoma due to acidic mucin that fills cytoplasm and nucleus displaces [1].

Pathohistological Analysis: Until 2003, PSRCBC was placed under 'mucin-producing carcinomas' and separated from both infiltrating ductal and lobular carcinomas by the World Health Organization (WHO) [19]. The histological examination of the neoplastic cells showed that they were small and round, with variable size-small to intermediate, scattered or funicular distribution, and with large intracytoplasmic mucin compressing the nuclei toward one pole of the cell $[3,20]$. Cytoplasm is abundant and may also be vacuolated [21]. In Figure 1 we present polymorphic signet ring cells (SRCs) with anisonucleosis and hyperchromasia. A nucleus pressed against the cell periphery as a result of diffusely filling the cytoplasm mucin. This occurs probably due to blockage in secretions due to deficiency of catenin, which probably occurs due to mutations [22, 23]. Periodic staining with Schiff (PAS) was performed and the cytoplasm showed PAS positivity suggesting the presence of intracytoplasmic mucin (Figure 4). The nuclei are large, irregular and with coarse chromatin. Characteristic of invasive ductal breast carcinoma (IDBC) with signet-ring cell differentiation is hypercellularity with single SRCs with a high nuclear grade (G3) and tubule formation [1]. Clinically, PSRCBC is defined as the presence of more than $20 \%$ of the SRCs in the tumor $[19,24,25]$. In our clinical case we report over $90 \%$ SRCs, foci located in IDBC with a moderate degree of cell differentiation (G2) (Figure 1). A study of the clinicopathological features of 22 patients with breast carcinoma containing SRCs found that the carcinomas had different degrees of differentiation, which meant that the degree of differentiation was not necessarily G3 [26]. Depending on the subcellular localization of mucin, SRCs are classified into intracytoplasmic lumina (ICL) type cells with large ICL containing mucin and non-ICL type cells with amorphous cytoplasmic areas, diffusely dispersed with mucin [26, 27]. In breast cancer, a significant relationship has been reported between the level of MUC1 expression and / or the presence of cytoplasmic staining with circumferential membranous accentuation pattern and adverse clinicopathological parameters [26]. For the study of intracytoplasmic cell mucin, most protocols offer staining with Alcian blue ( $\mathrm{pH} 2.5$ ), followed by the PAS technique. After PAS staining, we evaluate the presented clinical case with signet-ringlike malignant cells of the non-ICL cytological type (Figure 4/C).

Immunohistochemical Analysis: In PSRCC, the IHC study requires the expression of a number of markers such as ER (Figure 2/A), PR (Figure 2/B), HER2 (Figure 2/C), GCDFP (specific marker for breast tumors) (Figure 3/B) and E-cadherin (a marker that distinguishes invasive ductal carcinoma of the breast from invasive lobular carcinoma) (Figure 3/A). In the presented clinical case, we found positive estrogen and progesterone hormonal status and negative HER2 status (Figure 2), factors that determine a relatively good prognosis in moderately differentiated invasive ductal carcinoma (IDC) of the breast. The positive IHC expression of GCDFP (Figure 3/B) proves the primary breast carcinoma origin [3].

Differential Diagnosis: In mucinous breast cancer (MBC), different morphology and biological behavior compared to invasive ductal and lobular carcinoma of the breast, producing mucin were observed [28]. PSRCBC is characterized by numerous cells containing intracytoplasmic mucin and associated with aggressive clinical behavior, as opposed to extracellular mucin and favorable prognosis of MBC [12, 16, 17, 29-33]. Differential diagnosis with signet ring cell variant of lobular carcinoma, in which usually classic lobular component present, no DCIS, ER + and E-cadherin negative, is required [1].

Immunohistochemical differential diagnosis (DD): SRCBC can be divided into primary and metastatic tumors [3]. To differentiate between PSRCBC from metastatic SRCCs from different organs, Gross cystic disease fluid protein-15 (GCPFP-15) can be used as a sensitive marker of SRCBC and is useful even as an adjunct tool to diagnose metastatic SRCC of mammary origin [34]. SRCBCs are generally immunohistochemically positive for GCDFP-15, whereas SRCCs of the gastrointestinal tract are negative [34]. The positive expression of E-cadherin (Figure $3 / \mathrm{A}$ ) proves that it is an invasive ductal breast carcinoma with SRC differentiation and not an invasive lobular breast carcinoma. Furthermore, adenocarcinomas of the breast, stomach and colon show different CK7 and CK20 expression patterns [35-37]. Negative IHC expression of TTF1 (Figure 5/A) distinguishes primary breast cancer from lung adenocarcinoma and thyroid carcinoma. Negative IHC expression of CDX2 (Figure 5/B) differentiates primary breast cancer from colorectal cancer and negative expression of CK 20 (Figure 5/C) from gastrointestinal tumors. Expression of CK7, CK8, CK18 and CK19 was observed in more than $90 \%$ of all breast carcinomas, confirming their efficacy in immunohistochemical identification 
of breast cancer. CK20 expression was found more frequently than reported in previous studies, might constitute an indicator of poor prognosis and may be associated with the molecular apocrine subtype [38]. The DD of colorectal carcinomas requires an IHC analysis of CK7 and CK20, which detects CK7 negative expression and CK20 positive expression [39]. In the presented clinical case, we report a diffusely strong CK7 reaction in the tumor cells (Figure $3 / \mathrm{C}$ ) and a negative CK20 IHC expression (Figure $5 / \mathrm{C}$ ), which proves the primary origin of the carcinoma, namely from the mammary gland.

Prognosis: Generally, a significant number of SRCs, are associated with a worse prognosis $[40,41]$. SRCBC is a mucin producing neoplasm, and it can originate from both invasive lobular carcinoma and infiltrating ductal carcinoma, but more often from invasive lobular carcinomas [19, 34, 42]. Pure primary SRCC of the breast is a very rare tumor [20]. SRC differentiation in invasive ductal breast carcinoma (IDBC) is rare and associated with a high histologic grade (G3). Lymph node metastasis and distant metastasis are common, indicating an aggressive clinical behavior [15]. It is important to distinguish PSRCBC from MBC, since it is believed that treatment options and the clinical outcome are different [29]. Retrospective comparative analysis confirms the more aggressive behavior of PSRCBC compared to MBC. Patients with PSRCBC were presented with higher Ki67 labeling index, more advanced stages of disease and more frequent lymphatic metastasis at diagnosis than those with MBC [28]. It has been concluded that majority of $\mathrm{MBC}$ is positive for hormone receptor, which is associated with better prognosis [33, 43]. In general, the prognosis of breast cancer (BC) depends on a combination of a number of factors. Significant risk factors for distant metastases after complex treatment of $\mathrm{BC}$ were demonstrated: tumor $2-3 \mathrm{~cm}$ in diameter, G3, pN1, negative hormonal status, positive HER2 status, tumor necrosis, tumor invasion in blood vessels and tumor metaplasia [44]. In the presented clinical case, it is an advanced IDBC that has not been treated for one year. After the operation, metastatic axillary lymph nodes were identified, which did not allow radical lymphatic dissection at axillary level II and III, due to infiltration of the axillary vein. After 6 courses of systemic adjuvant $\mathrm{Ch}$, RT of the chest wall and regional lymph nodes with TD 46 Gy and antiestrogenic hormone therapy, we report 20 months disease-free survival with good quality of life.

\section{Conclusion}

Primary signet ring cell breast carcinoma is a rarely diagnosed malignant tumor. The diagnosis and the differential diagnosis of this rare neoplasm require precise pathohistological and immunohistochemical analysis. The prognosis in PSRCBC depends on the presence of significant risk factors for distant metastases: tumor $2-3 \mathrm{~cm}$ in diameter, G3, tumor necrosis, tumor invasion in blood vessels and tumor metaplasia, pN1, negative hormonal status and positive HER2 status. Complex treatment depends on the clinical stage, hormonal and HER2 status. After the complex treatment in locally advanced primary signet ring cell carcinoma of the breast with moderately differentiation, we achieve long-term local tumor control without distant metastases for nearly two years.

\section{References}

1. Roychowdhury M (2021) Signet ring cell. Pathology Outlines. com website. https://www.pathologyoutlines.com/topic/ breastmalignantsignetringcell.html.

2. El-Zimaity HM, Itani K, Graham DY (1997) Early diagnosis of signet ring cell carcinoma of the stomach: role of the Genta stain. J Clin Pathol 50: 867-868.

3. Xing Li, Yan-fen Feng, Wei-dong Wei (2013) Signet-ring cell carcinoma of the breast: a case report. World J Surg Oncol 11: 183 .

4. Benesch M, Mathieson A (2020) Epidemiology of Signet Ring Cell Adenocarcinomas. Cancers 12: 1544.

5. Leong FJ, Leong AS, Swift J (1996) Signet-ring carcinoma of the prostate. Pathol Res Pract 192: 1232-1241.

6. Jain V, Gupta K, Kudva R, Rodrigues GS (2006) A case of ovarian metastasis of gallbladder carcinoma simulating primary ovarian neoplasm: diagnostic pitfalls and review of literature. Int J Gynecol Cancer 1: 319-321.

7. Makino T, Tsujinaka T, Mishima H (2006) Primary signet-ring cell carcinoma of the colon and rectum: report of eight cases and review of 154 Japanese cases. Hepatogastroenterology 53: 845-849.

8. Michal M, Hes O, Kazakov DV (2005) Primary signet-ring stromal tumor of the testis. Virchows Arch 447: 107-110.

9. Belli S, Aytac H O, Karagulle E (2014) Outcomes of Surgical Treatment of Primary Signet Ring Cell Carcinoma of the Colon and Rectum: 22 Cases Reviewed With Literature. International Surgery 99: 691-698.

10. Saphir O (1941) Mucinous carcinoma of the breast. Surg Gynecol Obstet 72: 908-914.

11. Liu SM, Chen DR (2000) Signet-ring cell carcinoma of the breast. Pathol Int 50: 67-70.

12. Harris M, Wells S, Vasudev KS (1978) Primary signet cell carcinoma of the breast. Histopathology 11: 171-176.

13. Karabagli P, Kilic H (2010) Primary pure signet cell carcinoma of the breast: A case report and review of the literature. Breast Cancer 17: 1-4.

14. Kunisaki C, Shimizu S, Kato Y (1987) A case of primary signet ring cell carcinoma of the breast. Gan No Rinsho 11: 955-960.

15. Debajyoti Chatterjee, Amanjit Bal, Ashim Das (2017) Invasive Duct Carcinoma of the Breast with Dominant SignetRing Cell Differentiation: A Microsatellite Stable Tumor With Aggressive Behavior. Appl Immunohistochem Mol Morphol 25: 720-724.

16. Hull MT, Seo IS, Battersby JS, Csicsko JF (1980) Signet-ring cell carcinoma of the breast: a clinicopathologic study of 24 cases. Am J Clin Pathol 11: 31-35.

17. Steinbrecher JS, Silverberg SG (1976) Signet-ring cell carcinoma of the breast. The mucinous variant of infiltrating lobular carcinoma? Cancer 11: 828-840.

18. Naoto Kuroda, Nokiaki Fujishima, Masahiko Ohara (2007) Invasive ductal carcinoma of the breast with signet-ring cell and mucinous carcinoma components: diagnostic utility of immunocytochemistry of signet-ring cells in aspiration cytology materials. Diagn Cytopathol 35: 171-173.

19. Ellis V, Schnit S, Sastre-Garau X (2003) Invasive breast 
carcinoma. In: Tavassoli FA, Devilee P, editors. Tumors of the Breast and Female Genital System. Lyon: IARC; Invasive breast carcinoma 2003: 30-48.

20. Jashan Sandhu, VK Dubey, Manisha Makkar, Vijay Suri (2013) Pure primary signet ring cell carcinoma breast: A rare cytological diagnosis. J Cytol 30: 204-206.

21. Sauer T, Roskell D (2010) The breast. In: Gray W, Kocjan G, editors. Diagnostic Cytopathology. 3rd ed. UK: Churchill Livingstone Elsevier 2010: 213-214.

22. Frost AR, Terahata S, Yeh IT (1995) The significance of signet ring cells in infiltrating lobular carcinoma of the breast. Arch Pathol Lab Med 119: 64-68.

23. Maeno Y, Moroi S, Nagashima H (1999) Alpha-catenindeficient F9 cells differentiate into signet ring cells. Am J Pathol 154: 1323-1328.

24. Liu S-M, Chen D-R (2000) Signet ring cell carcinoma of the breast. Case report. Pathol Int 50: 67-70.

25. Frost AR, Terahata S, Yeh IT (1995) The significance of signet ring cells in infiltrating lobular carcinoma of the breast. Arch Pathol Lab Med 119: 64-72.

26. Ryuji Ohashi, Ayako Hayama, Keiko Yanagihara (2016) Prognostic significance of mucin expression profiles in breast carcinoma with signet ring cells: a clinicopathological study. Diagn Pathol 11: 131.

27. M Kamiya, K Mizuguchi, M Yoshimoto (1998) Cytologic diagnosis of signet-ring cell carcinoma of the breast. Acta Cytol 42: 650-656.

28. Wu X, Zhang Z, Li X (2016) Poorer Prognosis of Primary Signet-Ring Cell Carcinoma of the Breast Compared with Mucinous Carcinoma. PLoS ONE 11: e0162088.

29. Merino MJ, Livolsi VA (1981) Signet-ring carcinoma of the female breast: a clinicopathologic analysis of twenty-four cases. Cancer 48: 1830-1837.

30. Sandhu J, Dubey VK, Makkar M, Suri V (2013) Pure primary signet ring cell carcinoma breast: A rare cytological diagnosis. J Cytol 30: 204-206.

31. Karabagli P, Kilic H (2013) Primary pure signet cell carcinoma of the breast: a case report and review of the literature. Breast Cancer 20: 363-369.

32. Di Saverio S, Gutierrez J, Avisar E (2008) A retrospective review with long term follow up of 11,400 cases of pure mucinous breast carcinoma. Breast Cancer Res Treat 111: 541-547.

33. Zhang M, Teng XD, Guo XX (2014) Clinicopathological characteristics and prognosis of mucinous breast carcinoma. J Cancer Res Clin Oncol 140: 265-269.

34. Raju U, Ma CK, Shaw A (1993) Signet ring variant of lobular carcinoma of the breast: A clinicopathologic and immunohistochemical study. Mod Pathol 6: 516-520.

35. Chu PG, Wu E, Weiss LM (2000) Cytokeratin 7 and cytokeratin 20 expression in epithelial neoplasms: a survey of 435 cases. Mod Pathol 11: 962-972.

36. Wang NP, Zee S, Zarbo RJ (1995) Coordinate expression of cytokeratins 7 and 20 defines unique subsets of carcinomas. Appl Immunohistochem 11: 99-107.

37. Tot T (2000) The role of cytokeratins 20 and 7 and estrogen receptor analysis in separation of metastatic lobular carcinoma of the breast and metastatic signet ring cell carcinoma of the gastrointestinal tract. APMIS 11: 467-472.

38. Shao MM, Chan SK, Yu AMC (2012) Keratin expression in breast cancers. Virchows Arch 461: 313-322.

39. Ormsby A, Haskell R, Jones D (2000) Primary Seminal Vesicle Carcinoma: An Immunohistochemical Analysis of Four Cases. Mod Pathol 13: 46-51.

40. Lee WS, Chun HK, Lee WY (2007) Treatment outcomes in patients with signet ring cell carcinoma of the colorectum. Am J Surg 194: 294-298.

41. Cabebe EC, Mehta VK, Fisher G (2007) Gastric Cancer. eMedicine.com. URL: http://www.emedicine.com/med/ topic845.htm.

42. Fadare O (2006) Pleomorphic lobular carcinoma in situ of the breast composed almost entirely of signet ring cells. Pathol Int 56: 683-687.

43. Jensen EV (1966) Mechanism of estrogen action in relation to carcinogenesis. Proc Can Cancer Conf 6: 143-208.

44. Lena Petkova Marinova (2000) Radiation therapy after conservative surgery for early breast cancer. Dissertation for awarding the educational and scientific degree "Doctor" 2000: 50-77.
Copyright: (02021 Lena Marinovaz, et al. This is an open-access article distributed under the terms of the Creative Commons Attribution License, which permits unrestricted use, distribution, and reproduction in any medium, provided the original author and source are credited. 